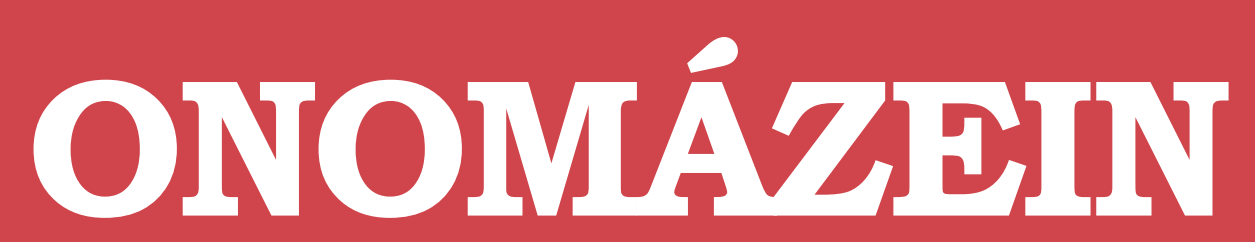

Revista de lingüística, filología y traducción
PONTIFICIA UNIVERSIDAD

\title{
Étude syntaxique et textuelle des constructions infinitives indépendantes
}

syntactic and textual analysis of independent infinitives

\section{Sara Quintero Ramírez}

Universidad de Guadalajara México

\section{(c) $($ i $)$}

Sara Quintero Ramírez: Departamento de Lenguas Modernas, Universidad de Guadalajara, México. | Correo electrónico: qsara@hotmail.com 


\section{Résumé}

Dans cette étude, nous présentons une analyse syntaxique et textuelle des constructions infinitives dites indépendantes telles que l'infinitif interrogatif, l'infinitif exclamatif, l'infinitif jussif et l'infinitif de narration. L'objectif de cette contribution est de montrer quelles sont les fonctions qu'assument ces constructions au niveau de la phrase et au niveau du texte. Dans le cadre de cette recherche, nous observons que même si ces quatre infinitifs sont considérés comme indépendants, pour leur entière compréhension, il faut tenir compte du cotexte et du contexte où ils sont employés.

Mots clés: infinitifs indépendants ; analyse syntaxique; analyse textuelle.

\section{Abstract}

This study undertakes a syntactic and textual analysis of independent infinitives in French, i.e., interrogative infinitives, exclamatory infinitives, injunctive infinitives and narrative infinitives. The main purpose of this research is to illustrate the functions of these infinitives at the sentence level and at the textual level. Although these infinitives are considered independent constructions, it is necessary to take into account cotext and context so that users of the language can understand them in a more comprehensive way.

Keywords: independent infinitives; syntactic analysis; textual analysis. 


\section{Infinitif français : traits généraux}

L’objectif de cet article est de dévoiler les fonctions qu’assument les infinitifs indépendants au niveau de la phrase et au niveau du texte. Pour ce faire, nou sélucidons, d’abord, les traits généraux de l'infinitifen français et des infinitifs indépendants. Deuxièmement, nous expliquons comment nous avons constituéle corpus de notre étude et comment nous avons procédé pour l'examiner. Troisièmement, nous présentons l'analyse. Enfin, nous exposons les conclusions que nous avons tirées de cetteétude.

L'infinitif, avec le participe et le gérondif, appartient aux formes verbales non finies. Au niveau syntaxique, l'infinitif français garde sa construction verbale, c'est-à-dire qu'il se comporte comme une forme verbale finie qui peut régir toute sorte de compléments verbaux (Sandfeld, 1965 : 3), à savoir : des compléments d'objet direct, des compléments d'objet indirect, des compléments circonstanciels de lieu, de temps, de manière, de but, de conséquence, de concession, etc. L'infinitif suivi de compléments verbaux se constitue en syntagme infinitif, et ce syntagme peut assumer une fonction nominale dans le cadre de la phrase. En effet, le syntagme infinitif peut jouer le rôle de complément verbal, de complément de nom, de complément de pronom, de complément d’adjectif, ou il peut même être utilisé dans une subordonnée relative et dans une interrogative indirecte (Lablanche, 2007 : 29).

\section{Constructions infinitives indépendantes}

Dans le cadre des infinitifs dans des propositions indépendantes, l'infinitif dévoile sa fonction la plus verbale. En d'autres termes, il ne joue plus une fonction nominale comme centre de proposition infinitive, mais une fonction nettement verbale qui peut se comparer avec celle que présentent les verbes finis dans une diversité de contextes. Ceci étant dit, la construction infinitive indépendante peut avoir le «même statut que la proposition grammaticale qui a pour centre la forme verbale personnelle» (Rémi-Giraud, 1988: 47). Dans ces constructions, l'infinitif assume la fonction de prédicat de la proposition et il apporte une information inédite. Autrement dit, l'infinitif se présente comme le nœud verbal de la construction indépendante (Riegel et al., 2001:334); par conséquent, il ne dépend pas d’une forme finie qui fonctionne comme pivot de la construction verbale.

Selon Denis \& Sancier-Chateau (1994: 293-294), Rémi-Giraud (1988:48), Turner (2000:3-34), Riegel et al. (2001:334) et Grevisse \& Goose (2005:1275-1276), il y a quatre types de constructions infinitives indépendantes : a) infinitif interrogatif, b) infinitif exclamatif, c) infinitif injonctif et d) infinitif de narration ${ }^{1}$. Les quatre constructions comportent un syntagme verbal

1 Quintero (2014b) analyse une cinquième construction infinitive indépendante : infinitif performatif. Néanmoins, dans cette étude nous n'en parlerons pas. 
constitué autour d'un infinitif présent. Dans ces constructions, « l'infinitif équivaut au mode personnel correspondant. II présente l'idée verbale et doit s'appuyer sur un contexte linguistique ou situationnel pour prendre sa valeur temporelle » (Riegel et al., 2001 : 335). Comme l'infinitif ne peut pas actualiser le procès, il n'a aucune valeur modale. "Cette absence de spécificité explique que l'infinitif puisse, selon les contextes particuliers, se plier à toutes les modalités: on le rencontrera donc dans divers emplois, où il pourra commuter avec d'autres modes - auxquels une valeur modale propre est normalement attachée » (Denis \& Sancier-Chateau, 1994: 293).

\subsection{Infinitif de narration}

L'infinitif de narration, appelé aussi infinitif historique2, se situe dans le cadre des constructions infinitives indépendantes (Riegel et al., 2001 : 335 ; Turner, 2000 : 3 ; Grevisse \& Goose, 2005 : 1275), car celle-ci ne dépend pas d'autres formes verbales au niveau syntaxique. Néanmoins, cette indépendance comporte certaines restrictions, car afin de comprendre adéquatement le sens et la fonction de cet infinitif, nous sommes obligés de considérer le cotexte antérieur (Quintero, 2014a : 4). En d'autres termes, «la phrase où il [l'infinitif de narration] figure ne se suffit pas à elle-même, mais elle s'insère dans une séquence d'actions, en se rattachant à la phrase précédente par et » (Riegel et al., 2001 : 335), comme on peut le vérifier à travers l'exemple (1).

(1) Au plus profond de l'œil d'un cyclope, il en fut un, et un seul, qui resta le même : « Personne, je suis. » se dit-Il pour lui-même. De je à Persona, parlons-nous à quatre? Oui. Yin et Yang. Mais dans quel hexagramme du Tao ? Le Dragon. Dans un avion l'air gonflait ses poumons : « Enfant, lui dit-on, mange ton riz. »Et lui de sourire comme le dernier Lama devant son mandala le jour de l'hymen de son karma. (Fayet, P. 2009. Attentat à Persona. Paris : Publibook)

L'infinitif de narration n'est analysé que très superficiellement dans les grammaires du français et dans les monographies de l'infinitif, car certains experts le considèrent comme une forme archaïque. D’une part, Huot (1981: 147) signale : «Bien que toutes les grammaires le mentionnent, nous ne pensons pas que l'infinitif historique ou de narration soit aujourd'hui vraiment productif en français. Et c'est volontairement que nous n'en donnons pas d'exemple.» D’autre part, Riegel et al. (2001:335) avertissent que l'infinitif de narration était utilisé habituellement au XVIIe siècle, mais qu'à l'heure actuelle il est très contraint et limité à certains textes, particulièrement de typologie narrative. Bien qu'au présent l'infinitif de narration ne soit pas tellement employé qu'au XVIIe siècle, on le trouve dans certains genres textuels très spéci-

2 Selon Englebert (1998: 76), c'est par le parallélisme qui existe entre le fonctionnement de l'infinitif de narration et celui du présent historique que certains préfèrent l'appeler d'une manière comparable à ce dernier. 
fiques, tel que des articles de presse, des histoires drôles, des récits personnels, des blogs et des forums sur Internet, entre autres (Quintero, 2015 : 271), comme nous le constaterons plus bas.

\subsection{Infinitif injonctif}

L'infinitif qui sert à exprimer des ordres, des conseils ou des instructions est désigné sous le nom d'infinitif injonctif ou infinitif jussif. Selon Riegel et al. (2001:335) et Denis \& Sancier-Chateau (1994: 294), cet infinitif constitue une sorte de variante de l'impératif étant donné qu'il a le même objectif de dire de faire ou de dire comment faire (Adam, 2001). Néanmoins, quelle est donc la différence entre l'impératif et l'infinitif injonctif ? Pour y répondre, Turner (2000: 12) signale qu'il y a des différences très nettes entre les deux. En effet, si on oppose l'infinitif injonctif à l'impératif, on peut observer qu'à partir d'une perspective morphologique, le premier ne porte aucune marque de personne, donc le lecteur, quel qu'il soit, devient le récepteur potentiel des injonctions, comme on peut observer en (2), où toutes les indications se présentent à l'infinitif; tandis que l'impératif en porte une bien claire, soit à la deuxième personne du singulier ou du pluriel, soit à la première personne du pluriel. En (3), on peut apprécier les indications d'une recette de cuisine qui sont toutes proposées à la deuxième personne (vous).

(2) Une base d'oignons, d'huile d'olive ou de beurre dans une cocotte à fond épais commence la recette. Une fois les oignons réduits, ajouter le riz (longs grains de préférence), et bien enrober les grains de la matière grasse jusqu'à les rendre translucides. Compter alors deux doses d'eau bouillante salée pour une dose de riz, et la verser sur le riz. Couvrir hermétiquement sur feux doux pendant 10 minutes. Ôter du feu, et laisser les grains finir d'absorber le liquide durant 5 minutes. Égrainer le riz à la fourchette, et à vous les plats d'inspiration exotique. (Le Journal des Femmes: «Faire cuire le riz méthode pilaf: un max de goût», 19/11/2014)

(3) Ne faites pas cuire le riz avec le sucre sinon celui-ci caramélise et il est difficile de contrôler la cuisson. De plus, le riz n'aura plus sa couleur crème.

Pour une facilité optimale, faites cuire le riz au micro-ondes, puissance moyenne, pendant 40 min et en surveillant la cuisson toutes les 10 min.

Mettez une platine à préchauffer au four. (Tordoir, Sophie, 2005 : Douceurs : La pâtisserie de Sophie Tordoir au Royal Hotel Windsor. Tielt: Racine Lannoo)

Au niveau de leur fonction dans la langue, Turner (2000: 12) affirme qu'à l'heure actuelle, l'impératif est utilisé à l'oral et à l'écrit; alors que l'infinitif injonctif est employé exclusivement dans la langue écrite. Ceci implique que le locuteur qui utilise un infinitif injonctif ne peut pas bénéficier des éléments extra-linguistiques des situations orales prototypiques (comme montrer du doigt le sujet). Par conséquent, celui qui utilise un infinitif injonctif dans un texte ne peut pas s'adresser à un ou à des co-locuteurs précis, puisqu'ils ne sont pas présents dans le contexte situationnel. 
De même, l'infinitif s'emploie en tant qu'injonction pour exprimer un ordre général et impersonnel (Grevisse \& Goose, 2005 : 1276). «Par conséquent, tout ‘lecteur', qui est donc un sujet potentiel du procès, s'identifie à ce sujet et nous arrivons à l'interprétation de l'énoncé en tant qu'injonction 'de portée générale’» (Turner, 2000 : 13). Cette portée semble convenir parfaitement en tant qu'ordre, conseil ou instruction dans des genres textuels très spécifiques, tels que des modes d'emploi, des recettes, des dépliants publicitaires, etc., où la série d'actions est présentée dans le texte sans qu'il y ait un rapport direct entre l'auteur du texte et un récepteur précis. En d’autres termes, il n’y a aucun sujet précis qui soit visé comme récepteur du texte; néanmoins, le co-énonciateur, plus particulièrement le lecteur quel qu’il soit, constitue un sujet potentiel pour remplir ce rôle (Turner, 2000 : 15).

\subsection{Infinitif interrogatif}

Dans le cadre des infinitifs interrogatifs, il y a deux types de constructions, l'une qui juxtapose le syntagme infinitif avec un pronom interrogatif et l'autre qui présente le syntagme infinitif sans se faire accompagner de pronom interrogatif (Turner, 2000). Cet infinitif dans ses deux modalités pourrait commuter avec le mode indicatif, même s'il n'exprime du procès que l'idée, et ne marque ni présent, ni futur, ni passé. « Mais sa virtualité lui permet de présenter seulement l'idée générale du procès, sans même évoquer la possibilité effective. L'énonciateur en reste à sa simple évocation »(Denis \& Sancier-Chateau, 1994: 293).

Les infinitifs interrogatifs du premier type sont formés du syntagme infinitif et d'un pronom interrogatif quelconque; ce dernier peut précéder ou suivre le syntagme infinitif selon le cas (Turner, 2000 : 19). Les exemples (4) et (5) montrent l'utilisation d'infinitifs interrogatifs comme titres de deux textes différents. Le premier exemple présente un syntagme infinitif, formé de l'infinitif choisir et du complément d'objet direct un dentifrice naturel, qui est précédé du pronom interrogatif pourquoi, le tout figurant comme le titre principal d'un reportage du magazine Marie Claire. Le deuxième exemple montre l'infinitif manger suivi du pronom interrogatif quoi; cette construction fait partie d'un titre d'un texte publié sur un site d'information générale sur le bien-être et la santé.

(4) Pourquoi choisir un dentifrice naturel?

Le brossage des dents fait partie de l'hygiène corporelle de base. Une bonne hygiène bucco-dentaire permet de garder des dents saines et d'afficher un joli sourire. Cependant, les dentifrices conventionnels sont controversés. Il existe dorénavant des dentifrices estampillés biologiques ou naturels. Quels sont leurs bénéfices? (Marie Claire : Pourquoi choisir un dentifrice naturel? 19/10/2015).

(5) Ventre gonflé : Manger quoi?

En période de crise de ventre ballonné, ou de ventre gonflé, il est impératif de passer en mode " d'épargne digestive », c'est-à-dire d'adopter des aliments (et des modes de cuisson) qui se digèrent facilement, ne fermentent pas. Alors en pratique, il faut manger quoi ? (Evrard, Nicolas, 24 février 2015 : «Ventre gonflé: Manger quoi ?», Onmeda, Santé, Bien-être, innovation). 
Le deuxième type de construction est composé du syntagme infinitif seul sous forme de question. Parfois, le syntagme infinitif peut se faire accompagner d'un pronom personnel qui prend la forme tonique. Ce type d'infinitif interrogatif s'identifie dans les textes écrits par la présence d'un point d'interrogation, et dans les textes oraux par le schéma prosodique interrogatif (Turner, 2000 : 4). L'exemple (6) montre trois infinitifs interrogatifs, les deux premiers forment une seule interrogation appartenant au deuxième type de construction, car ils ne se font accompagner d'aucun pronom interrogatif ; tandis que le troisième infinitif appartient au premier type parce qu'il est précédé par le pronom interrogatif comment. Les deux interrogations figurent comme titre d'un article scientifique.

(6) Faire ou ne pas faire de dictées? ou Comment faire de la dictée un moment d'apprentissage? La dictée, cet exercice qui n'a d'existence qu'à l'école, est plébiscité par de nombreux parents, en particulier dans les milieux populaires, pour lesquels apprendre exige de savoir écouter, d'être sage, d’obéir au Maître. (Bernardin Jean. 2005 : «Apprendre ensemble, réussir ensemble », GFEN, DIALOGUE 118)

Dans les deux types de constructions infinitives interrogatives, les infinitifs n'ont pas un sujet syntaxique exprimé; de telle sorte que le sujet peut être considéré comme un agent indéfini générique.

\subsection{Infinitif exclamatif}

L'infinitif exclamatif exprime un sentiment vif tel que le souhait, le regret, l'indignation, la surprise, etc. Cette construction infinitive peut s'employer avec un sujet exprimé (7) ou sans sujet explicite (8). Quand le sujet est exprimé, celui-ci prend sa forme tonique et se sépare de l'infinitif à travers une pause à l'oral ou une virgule à l'écrit. Quand il n'y a pas de sujet explicite, son interprétation peut rester générale ou, en tout cas, le sujet peut se récupérer du cotexte. «L'absence de forme conjuguée et l'absence du sujet (ou sa nette séparation d'avec l'infinitif quand il est exprimé) permettent de présenter l'idée du procès à l'état brut; sa seule évocation dans la situation donnée suffit à indiquer le sentiment éprouvé » (Riegel et al., 2001: 335). En d’autres mots, le procès n'est pas actualisé, il n'est l'objet que d'une évocation (Denis \& Sancier-Chateau, 1994 : 294).

(7) Sœurette,

Tu sais que j'ai quelques problèmes de respiration alors je suis partie chez le médecin et je ferai les courses. Prends ma carte de crédit et va faire du shopping car il faut profiter de Paris! Ta Sœur qui t’aime Sofia.

Je suis tellement émue. Elle, écrire une chose pareille, elle est pro! Je prends mon petit déjeuner et prends la carte pour faire du shopping.

(https://www.wattpad.com/100178479-jumelles-chapitre-1) 
(8) Voyager en pays musulman!

Je suis exactement le genre de voyageuse qui n'aime pas avoir l'air d'une touriste. J'essaie de me fondre parmi les locaux. Je n'aime pas me faire regarder. Je n'aime pas attirer l'attention. Je sens toujours que le mot «touriste » est écrit dans mon front. J'essaie toujours d'apprendre quelques mots de la langue locale avant de partir. Et je dois admettre que mon teint basané est un atout de taille pour passer inaperçue dans beaucoup de pays. (http://voyagerenfamille.com/: 20/10/2012)

\section{Méthodologie}

\subsection{Formation du corpus}

Pour cette recherche, nous avons élaboré un corpus de 250 textes. Pour ce faire, nous avons procédé en deux étapes. Dans une première étape, nous avons examiné 150 textes de différentes typologies et de différents genres. En effet, nous avons recueilli 30 textes de presse écrite (des reportages, des chroniques, des critiques, etc.), 20 textes de magazines (des articles, des courriers des lecteurs, des horoscopes, etc.), 15 textes littéraires (des extraits de romans, des contes et des poèmes), 20 textes académiques (des articles spécialisés, des extraits de thèses, des conférences magistrales, des ateliers académiques, etc.), 15 textes télévisés (des journaux télévisés, des interviews et des extraits de films), 30 textes d'Internet (des courriers électroniques, des blogs, des forums, des mèmes, etc.) et, enfin, 20 textes élaborés dans des situations formelles (des discours, des débats et des conférences de presse) $)^{3}$.

Cette première étape nous a permis de reconnaître les genres textuels où étaient utilisés les infinitifs indépendants. Dans une deuxième phase, nous avons fait une recherche plus exhaustive de ces infinitifs mais seulement dans les textes où nous les avions localisés lors de la première phase. Pour ce deuxième temps de construction du corpus, nous avons fait une sélection de 100 autres textes. En fait, nous avons considéré exclusivement des textes de presse écrite (30), des textes de magazines (10), des textes littéraires (10), des textes d'Internet (30) et des textes académiques (20). Par conséquent, entre les deux phases de collecte de données nous avons pris en compte 250 textes différents.

\subsection{Procédé d'analyse}

Une fois le corpus constitué, nous y avons identifié les infinitifs indépendants dans les différents textes. Après avoir distingué les constructions infinitives, nous les avons examinées

3 Nous avons utilisé le corpus de cette première étape pour une autre recherche menée en 2014. 
suivant des critères syntaxiques et textuels. Tout d'abord, nous nous sommes focalisés sur les caractéristiques syntaxiques telles que la configuration dont sont produits les éléments linguistiques qui figurent dans le cotexte antérieur et postérieur des infinitifs. Ensuite, nous avons identifié les genres textuels et la situation communicative spécifiques où les différentes constructions indépendantes sont employées le plus. Enfin, nous avons observé le but communicatif de chaque construction infinitive indépendante.

\section{Analyse}

Dans cette section, nous exposerons les données les plus significatives de notre étude. D’une part, nous élucidons les caractéristiques syntaxiques et textuelles que nous avons observées à propos de chaque construction infinitive indépendante à partir de notre corpus. D’autre part, nous présentons des exemples d'infinitifs indépendants (sans correction) dans leur cotexte antérieur et postérieur afin de pouvoir les examiner à partir d'une perspective plus textuelle. Par conséquent, nous avons considéré le paragraphe comme unité d'exemplification.

\subsection{Infinitif de narration}

Avant d'examiner les traits textuels de l'infinitif de narration, il est convenable d'identifier ses caractéristiques fondamentales au niveau syntaxique. Comme on peut remarquer dans l'exemple (9), cette construction infinitive indépendante implique un syntagme infinitif (frapper en mesure, avec un parfait sentiment [...]) et un syntagme nominal (l'enfant), tous les deux mis en relation par la particule de, et le tout étant introduit par la conjonction et afin de constituer la configuration canonique suivante : conjonction et + syntagme nominal + de + syntagme infinitif (Riegel et al., 2001 ; Englebert, 1998 ; Melis, 2000 ; Grevisse \& Goose, 2005 ; Torterat, 2008).

(9) [I] I avait six ans lorsqu'une troupe de musiciens ambulants, passant par Montaigu, s'arrêta sur la place et se mit à jouer. Ce concert en plein vent le plongea dans le ravissement [...] Le chef, le plaçant sur un grand cheval chargé 'une paire de timbales, lui mit dans les mains les baguettes de l'instrument; et l'enfant de frapper en mesure, avec un parfait sentiment du rythme, au grand ébahissement des badauds. (Tiersot, Julien, 1903 [2015] : Histoire de la Marseillaise. Nombreuses gravures documentaires. Paris: Ed. Ligaran)

Néanmoins, cette distribution peut subir des changements, car le syntagme nominal et la conjonction peuvent changer de place. D'une part, le syntagme nominal peut suivre l'infinitif au lieu de le précéder, comme nous le constatons dans l'exemple (10), où le syntagme nominal, Jean-René Dufort, se place après l'infinitif dire. À propos de ceci, Stage (2006 : 90) signale 
que les exemples d'inversion entre le syntagme nominal et le syntagme infinitif ne sont pas très fréquents dans le français de France, mais plutôt dans le français de Belgique ou celui du Canada, comme c'est le cas de notre exemple.

(10) Guy Fournier a promis un humour gentil pour ce week-end des Gémeaux... On a eu droit tout de même à des textes de Richard Gohier un peu méchants, comme la comédie de Radio-Canada, les tumultueuses aventures de Gilles Gougeon; un message sur le gros porc frais de Normétal ; et on a invité les gens à regarder une image de Simon Durivage... Et de dire Jean-René Dufort : " C'est sûrement le plus de fois que le monde l'a regardé cette année... » On sait que son émission Aujourd'hui bat de l'aile. (Canoë, le Journal de Montréal : «Une avant-première des Gémeaux qui tombe à plat... », 23/11/2003)

De même, « quand il y a coréférence avec le sujet grammatical d'un verbe fini précédent ou entre le sujet logique d'un infinitif narratif et le sujet grammatical du verbe lié, le sujet peut être omis »(Stage, 2006 : 92), comme nous pouvons l'apprécier dans l'exemple (11), où la construction infinitive ne présente pas de sujet grammatical, car elle le récupère de la phrase précédente: cette habitante de la rue Maurice Prestaut qui ne décolère pas [...].

(11) D'aucuns pensent même que l'instauration de ce dispositif très discutable a rendu la voie plus dangereuse qu'avant... comme cette habitante de la rue Maurice Prestaut qui ne décolère pas: « On ne peut donc plus accéder directement à cette rue, on est obligé de faire un détour et d'emprunter le chemin des Ormes. On se retrouve alors dans une rue devenue à double sens unique. »Et d'ajouter : "Il n'est pas rare de voir les véhicules prendre le sens interdit car ils ne savent pas par où passer. » (Le Parisien : "Ille-et-Vilaine: Rennes invente la rue en double sens interdit $», 13 / 10 / 2015)$

D'autre part, la conjonction et peut faire place à un connecteur discursif tel que mais, alors, puis, entre autres (Englebert, 1998 : 64-65), afin de relier la construction infinitive avec celle qui la précède, comme on constate en (12), où le syntagme nominal, la particule de et le syntagme infinitif sont précédés du connecteur discursif mais; ce connecteur ne s'occupe pas seulement de relier la construction infinitive avec celle qui se conjugue dans un temps verbal fini et qui figure dans le cotexte antérieur, [o]n court après lui, on le ramène à la maison paternelle [...], mais s'occupe aussi de conférer à l'infinitif de narration une valeur d'opposition par rapport à la construction précédente. Enfin, il est rare mais possible que la conjonction et (ou un connecteur discursif la remplaçant) soit absente dans la construction infinitive, comme on peut le vérifier en (13), où les trois autres éléments constitutifs de l'infinitif de narration ne sont précédés d'aucune conjonction. On peut constater aussi que cet exemple présente l'inversion du syntagme nominal avec l'infinitif.

(12) Le jeu lui plut si bien que, lorsque les musiciens s’éloignèrent, il les suivit, toujours monté sur son grand cheval et tapant sur ses timbales. On court après lui, on le ramène à la maison paternelle: pleurs, reproches de la mère; mais lui de répondre : "Oh! maman, ils jouaient si bien du violon! » 
(Tiersot, Julien, 1903 [2015] : Histoire de la Marseillaise. Nombreuses gravures documentaires. Paris Ed. Ligaran)

(13) Les chercheurs essaient de comprendre comment l'inflammation s'installe, de dire le docteur Claude Asselin. Ce qu'on ne saisit pas dans ces maladies, c'est qu'il y a souvent des périodes de rémission. Les mécanismes de contrôle de l'inflammation présents chez la majorité des gens font peut-être défaut chez les individus souffrant de ces maladies-là. (Giguère, Elise, s/d : Les mystères de l'intestin. Université de Sherbrooke, Canada)

Nous venons de mentionner que la conjonction et ou les connecteurs qui apparaissent à sa place établissent un lien entre la construction infinitive narrative et le fragment textuel qui la précède. En effet, l'infinitif de narration produit un effet stylistique de vivacité (Denis \& Sancier-Chateau, 1994), de soudaineté (Riegel et al., 2001), de rapidité (El Kassas, 2007) entre deux actions, l'une (celle exprimée à travers la construction infinitive) qui découle inéluctablement d'actions antérieures (celles exprimées à travers des formes finies). C'est la raison pour laquelle l'infinitif de narration ne peut pas figurer en début de phrase; au contraire, il se situe à la suite d’une série de verbes exprimés à travers des formes finies.

Cet infinitif est employé dans une phrase déclarative ou assertive. De plus, les informations apportées par cette construction dans les exemples de notre corpus ressemblent, comme le signale Englebert (1998: 78), à celles présentées dans les didascalies, telles que le changement de locuteur ou la reprise de parole d'un même locuteur en marquant un statut différent entre ses interventions. Nous pouvons constater ce phénomène dans l'exemple (14), dans lequel l'auteur du texte, Puren, reprend le discours de Sanderson et présente, tout d'abord, une citation directe en l'introduisant par un présent historique : écrit. Puis, il utilise l'infinitif de narration pour paraphraser ce que Sanderson dit encore une fois, c'est-à-dire pour employer le style indirect. En d'autres termes, on observe que grâce à l'infinitif de narration, Puren peut mettre en relief la transition entre le style direct et le style indirect.

(14) E. Sanderson écrit ainsi dans la préface de son manuel d’anglais: «Il est souvent malaisé pour eux [...] l'enseignera en mettant, s'il le veut, un exemplaire dans les mains de chacun de ses élèves et en en retirant les corrigés. " Et l'auteur de garantir que les progrès des maîtres seront aussi sûrs et rapides que ceux de leurs élèves [...] (Puren, Christian, 1988 : Histoire des Méthodologies. Paris: Cle International)

Un autre type d'information qui est présentée par l'infinitif de narration consiste en des actions réalisées par les personnages du texte ou des attitudes prises par eux. Dans l'exemple (15), nous pouvons vérifier que les deux infinitifs de narration du texte font référence à une attitude prise par les personnages de l'histoire en raison de ce que vient de dire le personnage-narrateur.

(15) Lorsque je la vis, je dis: « Maman aussi, une fois, elle a fait un dessert au curaçao. » et je prononçais le second «a» dans le mot. Tous de s'esclaffer! « Mais on ne prononce pas comme ça. Faut sortir 
de ta campagne », «Du curaçao, insista Jeanne, pas curaçao en prononçant le deuxième «a »! Et tous de rire encore : "C'est les péquenots qui prononcent comme ça. » (Rousselet, Jules, 2014 : Réparations. Littératures.fr).

Englebert (1998: 79) explique que la fonction de l'infinitif de narration dans le cadre des chroniques de la presse francophone est d'assurer la distribution du discours rapporté afin de mettre en relief la transition entre les passages textuels qui ont un statut différent entre eux, ce qui souligne l'idée de soudaineté (Riegel et al., 2001) et de rapidité (El Kassas, 2007) dont nous avons parlé antérieurement. Ceci peut être vérifié dans l'exemple (16), dans lequel le fil conducteur du récit est le passé composé. Nous pouvons synthétiser le fragment textuel de la manière suivante : le personnage 1 explique le titre de son album, le personnage 2 pose une question à propos du titre, le personnage 1 intervient pour la deuxième fois, et c'est ici où l'auteur du texte décide d'utiliser l'infinitif de narration pour présenter cette deuxième intervention : et Carla de répondre [...]. Enfin, pour la deuxième intervention du personnage 2 , et la dernière construction verbale du passage textuel, l'auteur reprend le passé composé.

(16) Carla Bruni a dû auparavant en expliquer le titre à son auditoire britannique. "Ça parle d'une addiction à l'amour. Etre accro de quelqu'un de façon toxique et délicieuse à la fois. » L'animateur lui a alors demandé si elle l'avait déjà vécu. Et Carla de répondre : "Bien sûr, et vous-même ?» «Oui, en ce moment », a rétorqué Jools Holland, provoquant les rires. (20 minutes : "Carla Bruni : "La nuit je chante pour mon mari” », 17/09/2008)

\subsection{Infinitif injonctif}

D’une part, Turner (2000 : 15) et Grevisse \& Goose (2005: 1276) affirment que cet infinitif a une valeur d’ordre général et impersonnel. Voilà pourquoi le contexte situationnel des genres textuels où il apparaît joue un rôle important dans l'interprétation univoque d'un infinitif injonctif. D’autre part, Denis \& Sancier-Château (1994 : 294) signalent que cette construction est employée «lors que le destinataire de l'énoncé doit rester implicite, dans sa plus grande virtualité. » Selon El Hadi (2011: 201), cet infinitif fait une exhortation mais, en même temps, efface l'agent qui est censé réaliser l'action. Par conséquent, on le voit souvent dans des genres textuels fort spécifiques tels que : des recettes de cuisine, des dépliants publicitaires, des codes de la route, des modes d'emploi de produits divers, des prescriptions officielles et même des mémentos pour noter les choses à faire.

Les exemples de notre corpus relèvent pour la plupart de guides de produits divers, de notices de médicaments et de recettes de cuisine. Adam (2001 : 12) indique que les genres textuels de type injonctif visent un but totalement pratique car ils ont la fonction de faciliter et même d'aider le lecteur à réaliser une tâche qu'il a l'intention d'accomplir. 
Kabatek (2008 : 203) affirme que ces infinitifs sont clairement jussifs grâce à la situation de communication dévoilée dans le cadre de ces genres textuels où ils sont utilisés, car le récepteur du texte, c'est-à-dire la deuxième personne, cherche ces textes justement afin d'agir suivant les indications présentées. Adam (2001 : 13) ajoute que «le caractère d'obligation et l'échelle de contrainte de ces actes de discours jussifs varie [sic] d'un genre à un autre : la liberté de ne pas suivre l'injonction-recommandation est très faible pour tous les genres régulateurs (consignes et règlements), très grande pour les conseils et autres horoscopes, moyenne pour les genres procéduraux (recettes, guides, notices de montage) ». En (17), nous observons un fragment de guide où le lecteur est fortement conseillé de suivre les trois pas présentés dans le texte pour préparer une boisson SRO afin de contrôler la diarrhée et d'éviter des dangers tels que la perte de liquides et la malnutrition. L'injonction et l'interdiction des actions sont nécessaires pour obtenir le but désiré.

(17) Comment préparer une boisson à base de SRO?

1. Vider le contenu d'un sachet de SRO dans un récipient propre. Lire les instructions sur le sachet pour ajouter la quantité d'eau potable indiquée. Si l'on n'ajoute pas assez d'eau, on risque d'aggraver la diarrhée.

2. Ajouter uniquement de l'eau. Ne pas mélanger les SRO avec du lait, de la soupe, du jus de fruit ou des Boissons gazeuses. Ne pas rajouter de sucre.

3. Bien mélanger et donner à boire à l'enfant en utilisant une tasse propre. Ne pas utiliser de biberon. (Guide des Professionnels de la Santé)

Selon Adam (2001 : 12-13), les prédicats actionnels sont caractéristiques de ces genres textuels et les temps verbaux de ces prédicats actionnels, soit l'infinitif soit l'impératif, ont une fonction illocutoire fort importante. Ces prédicats peuvent être formés d'une seule action, de deux actions comme en (18), ou de plusieurs actions successives comme dans l'exemple (17) qui présente une série d'actions injonctives.

(18) 21 pilules : en prendre une chaque jour pendant 21 jours, puis cesser pendant sept jours (Guide d'utilisation des contraceptifs oraux)

Quand le texte ou le fragment textuel est constitué d'une série d'infinitifs injonctifs, Turner(2000 :14) affirme que ces infinitifs ont tendance à présenter une sorte de rupture avec ce qui précède, car quand les infinitifs sont conçus en série dans un texte précis, ceux-ci correspondent à une série de renvois à la notion, « ce qui implique que chaque procès est envisagé comme étant en rupture avec ce qui précède, les procès n’étant repérés les uns par rapport aux autres que dans la mesure où des procès globalisés et juxtaposés les uns aux autres peuvent acquérir la valeur de renvois à des occurrences par le fait de se trouver ainsi dans une suite chronologique ».

L'exemple (19) est formé de quatre infinitifs injonctifs : utiliser, verser, fouetter et vérifier. Chaque infinitif à valeur injonctive semble ne pas avoir un lien direct avec l'infinitif précé- 
dent car chaque construction infinitive constitue une étape concrète. Néanmoins, l'ensemble des étapes exprimées toutes à travers des infinitifs constitue le tout pour la préparation des blancs en neige.

(19) Préparation:

a. Utiliser des blancs sans jaune, ni coquille d'œuf.

b. Verser les blancs dans un récipient très propre.

c. Sur un rythme régulier, fouetter énergiquement en effectuant un geste circulaire pour incorporer le maximum d'air.

d. Quand les blancs restent accrochés au fouet, ils sont prêts.

e. Attention à ne pas trop battre les blancs au risque qu'ils ne grainent (formation de grains).

f. Vérifier que les blancs tiennent bien en retournant le récipient : ils doivent rester collés.

(http://www.marmiton.org/)

Enfin, dans notre corpus, nous observons des infinitifs injonctifs précédés de la prépositionà, tels que (20) et (21). Nous considérons4 que ces infinitifs peuvent être interprétés comme des ordres à une force obligatoire plus ferme que ceux qui ne sont pas précédés de préposition. Cette idée est renforcée par l'incompatibilité de ces constructions avec la formule d'atténuation s'il vous plait.

(20) La salade et l'oignon: Pas difficile, couper très fin des feuilles de salade et des oignons, à servir dans des plats séparés. (http://zaboo.canalblog.com/archives/2007/05/02/4810743.html)

(21) Voie d'administration et mode d'emploi

A prendre parvoie orale.

Les comprimés sont avalés avec un peu d'eau, sans les mâcher ou les couper, de préférence après le repas [...]. (Notice de Cardioaspirine $100 \mathrm{mg}$ comprimés gastro-résistants)

\subsection{Infinitif interrogatif}

L'infinitif interrogatif est connu aussi sous le nom d'infinitif délibératif car ces propositions interrogatives expriment surtout la délibération et la perplexité. Khodabocus (2014: 2258) signale qu'il s'agit d'un locuteur imprécis qui se pose des questions à lui-même, comme c'est le cas de (22). De même Riegel et al. (2001:335) avertissent: "Dans une phrase interrogative sans sujet exprimé, on peut employer l'infinitif délibératif [...] Le sujet est le locuteur ou un agent indéfini générique. Dans les monologues de théâtre, le personnage se pose une question à soimême. » En (22), le personnage se pose la question à lui-même et il y répond immédiatement.

4 De la même manière que nous interprétons en espagnol l'infinitivo yusivo précédé de la préposition a (Hernanz, 1999 : 2339). 
(22) Eh oui, le natto, comment dire, ça fait à peu près le même effet à un Français que, par exemple, le fromage de chèvre à un Japonais... C'est à dire que ça provoque d'abord une réaction d'incrédulité ("??? qu'est-ce???"), puis de doute ("ça se mange???"), parfois de résistance ("Moi, manger ça? Jamais!"), et plus rarement d'héroïsme matiné d'inconscience [...]. (http://oriibu.canalblog.com/ archives/2013/06/09/27362124.html)

Néanmoins, la délibération et la perplexité ne sont pas les seules valeurs communicatives de ces constructions infinitives. Dans notre corpus, nous avons réuni des exemples d'infinitifs interrogatifs qui sont accompagnés d'un pronom interrogatif. Ces constructions présentent des caractéristiques différentes à la délibération et surtout des fins communicatives bien distinctes. En effet, la plupart de nos exemples de syntagmes infinitifs précédés ou suivis d'un pronom interrogatif constituent des titres et des sous-titres de différents genres textuels, à savoir: des articles et des reportages de magazines, des manuels d'écriture, des notices de médicaments, etc. Dans l'exemple (23), nous observons un infinitif interrogatif qui constitue le sous-titre d'une notice de médicament. Ladite construction a le but de présenter un nouveau fragment textuel au lecteur et de lui donner ensuite une série de recommandations à suivre afin qu’il prenne adéquatement le médicament.

(23) Comment prendre Cardioaspirine?

Veillez à toujours prendre ce médicament en suivant exactement les instructions de cette notice ou les indications de votre médecin ou pharmacien [...] (Notice de Cardioaspirine 100 mg comprimés gastro-résistants)

En (24), nous observons un autre exemple d'infinitif interrogatif dans un manuel d'écriture qui présente des fiches pour guider un auteur à bien rédiger différents genres textuels. La fiche que nous considérons pour le corpus est constituée d'une diversité de sous-titres rédigés sous forme de construction infinitive interrogative, comme celle que nous observons dans l'exemple (23). Dans le corps du texte, nous distinguons une série de constructions infinitives injonctives qui présentent au lecteur des recommandations à suivre. Dans au moins la moitié des exemples d'infinitifs interrogatifs de notre corpus, nous avons trouvé aussi des exemples d'infinitifs injonctifs. En effet, les infinitifs interrogatifs ont tendance à apparaître dans le titre ou les sous-titres du texte, tandis que les infinitifs injonctifs ont tendance à se présenter dans le corps du texte.

(24) Quelles explications donner?

Toute explication se fera selon deux plans complémentaires, l'un statique et l'autre dynamique.

- Désigner et décrire les différents éléments du mécanisme et leurs fonctions.

- Analyser ensuite, très rigoureusement, les étapes du fonctionnement. Il faut toujours s'efforcer de répondre à des questions simples : quoi ? où ? quand ? comment ? pourquoi ? Elles permettent de ne rien oublier. (Peyroutet, Claude, 2005 : La pratique de l'expression écrite. Paris: Nathan) 
Nous considérons que les infinitifs jussifs de nos exemples se chargent aussi de donner une réponse aux interrogations posées à travers des infinitifs interrogatifs au début du fragment textuel. Nos observations des exemples nous amènent à penser que les infinitifs interrogatifs qui constituent les titres et les sous-titres de différents exemples de notre corpus sont des questions que le lecteur pourrait se formuler lui-même, et que les infinitifs jussifs offrent la réponse-injonction attendue par le lecteur. Par conséquent, les constructions infinitives interrogatives de notre corpus pourraient être considérées comme des stratégies textuelles d'un auteur pour attirer l'attention du lecteur afin que celui-ci lise la suite du texte.

Enfin, dans notre corpus nous identifions aussi des infinitifs interrogatifs qui figurent dans le corps du texte, tel est le cas de l'exemple (25), où nous avertissons deux constructions infinitives interrogatives, à savoir: Comment s'engager dans la bonne voie? et comment se professionnaliser, rebondir après quelques années d'enseignement [...] ? La première construction interrogative se situe juste à la fin du premier paragraphe du fragment textuel, tandis que la deuxième construction interrogative (à quatre infinitifs) se situe juste au début du troisième paragraphe du fragment textuel.

(25) Décrié par les uns en raison de la précarité qu'on y trouve, 'chapellisé' par d'autres face à la raréfaction des situations confortables, encensé par ceux qui y ont trouvé épanouissement personnel et professionnel, le FLE est tout à la fois un cul-de-sac et une piste d'envol. Comment s'engager dans la bonne voie?

Peut-être en commençant par s'interroger sur les essentiels. Vos essentiels. Aventure et précarité ou cadre et sécurité? [...]

Bref, comment se professionnaliser, rebondir après quelques années d'enseignement, valoriser ses compétences ou encore panacher le FLE avec un autre champ professionnel?

Nous vous présentons pour cette rentrée 2013-2014 un dossier détaillé sur les métiers du FLE. [...] (Agence de promotion du FLE. Les métiers du FLE, 16/10/2013).

\subsection{Infinitif exclamatif}

Les infinitifs exclamatifs ressemblent considérablement, au niveau syntaxique, aux infinitifs interrogatifs du deuxième type (vid. supra), c'est-à-dire à ceux qui sont formés du syntagme infinitif sous forme de question ou à ceux qui juxtaposent le syntagme infinitif avec un pronom personnel qui prend sa forme tonique. «La seule différence entre l'énoncé 'interrogatif' et l'énoncé 'exclamatif' de ce type se trouve dans le schéma prosodique qui lui est donné, celui-ci étant signalé dans la typographie par la présence du point d'interrogation ou par le point d'exclamation » (Turner, 2000 : 10). Nous pouvons constater cette ressemblance entre ces deux constructions infinitives car l'infinitif interrogatif de (22) pourrait être interprété en tant qu'infinitif exclamatif si à l'oral, comme le suggère Turner, on modifiait le schéma prosodique d'interrogation à exclamation et, si au niveau écrit, au lieu d'écrire un point d'interrogation, on mettait un point d'exclamation. 
Comme dans le cadre des infinitifs interrogatifs, les infinitifs exclamatifs peuvent ne pas présenter de sujet explicite, comme c'est le cas de (26), où le sujet n'est pas exprimé, mais peut être récupéré dans le cotexte antérieur. L'auteur exprime ici l'indignation par le manque de professionnalisme des vendeurs de guitares. Dans notre exemple, l'auteur écrit trois points d'exclamation et un point d'interrogation. Nous considérons que comme il s'agit d'un forum sur Internet, l'auteur du texte peut se permettre de mélanger la ponctuation, car celle-ci n'est pas aussi stricte que dans d'autres genres textuels. En plus, comme signale Sandfeld (1965: 204), dans bien des cas d'infinitifs indépendants, l'exclamation est souvent interrogative.

(26) N’ayant pas de nouvelles après I semaine (la guitare à 2 semaines de retard), je téléphone et là on m’apprends que comme par magie la guitare n'existe plus et qu'ils l'ont commandé au canada. Et voilà je dois attendre encore un mois.

C'est du professionalisme ça? Me faire venir pour rien !!?! Les 2 semaine d'attentes se transforme en 1 mois et demi ?!!??! (http://www.guitariste.com/forums/bla-bla-et-guitare,coup-de-gueulecontre-musik-produktiv,178316.html)

Par ailleurs, il y a des cas où la construction infinitive exclamative ne présente pas de sujet explicite, car son interprétation se veut plus générale, comme nous pouvons le remarquer dans l'exemple (27), où l'infinitif exclamatif n'a pas de sujet exprimé. Nous considérons que le sujet est absent dans cette construction afin de montrer que la phrase où figure l'infinitif exclamatif est énoncée par beaucoup de parents qui ont des enfants, et que le lecteur peut se trouver dans une situation similaire et, par conséquent, s’identifier.

(27) «Ah! voyager avec des enfants! c'est épuisant!» Dixit de beaucoup de parents! Je réalise que beaucoup de mes amis ne voyagent plus dès qu'ils ont des enfants ou prennent soin de les laisser chez les grands-parents avant de filer en avion pour une semaine. Et vous, que préférez-vous [...] (http:// voyagerenfamille.com/: 28/07/2012)

Selon Sandfeld (1965: 203), dans le cadre des textes dialogiques, l'infinitif exclamatif a tendance à figurer dans les tours de parole qui répondent à une intervention antérieure. Dans la réponse, l'infinitif exclamatif reprend un verbe précédent, tel que (28), où le locuteur B emploie l'infinitif exclamatif pour montrer l'indignation et la surprise par rapport à la prédication énoncée par le locuteur A. Nous considérons que le locuteur B met en question et la prédication (t’aider) et le sujet de la prédication (moi).

(28) A: -Comment? As tu pris conscience que ce n'est pas un acte qu'il faut prendre à la légère !

B : - Oui, et tu vas m'aider.

A : - Moi t’aider! Je ne veux pas être complice de sa mort!

B : - Si, tu conduiras.

A : -D’accord mais je ne veux pas voir notre chat turboto se faire piquer.

(http://welovewords.com/documents/mort-annoncee) 
Dans notre corpus, nous inventorions des infinitifs exclamatifs avec la formule suivante: Et dire que + construction avec verbe fini (au mode indicatif) + point d'exclamation (!), tels que les exemples (29-31). Nous observons ces constructions spécifiques plus particulièrement dans des mèmes et des blogs sur Internet, comme on peut le constater dans nos exemples.

(29) Et dire qu'il y en a qui bossent !!! (http://www.memegen.fr/meme/cpık5w)

(30) Et dire qu'il faudra tenir jusqu'à la retraite... Pfff... J'ai pas le goût! (http://www.memegen.es/meme/ h6vyub)

(31) Et dire que j'ai pas pu venir !! :'( (http://www.memegen.com/meme/wvqnrh)

\section{Conclusions}

Tout au long de ce travail nous avons élucidé les facteurs syntaxiques et textuels qui caractérisent l'utilisation des quatre infinitifs indépendants en français. Bien que ces infinitifs soient considérés comme indépendants, nous avons averti que pour leur entière compréhension, il faut tenir compte du cotexte et du contexte où ils sont employés. Nous présentons maintenant une synthèse de leurs traits les plus importants:

Infinitif de narration: D’après les observations du corpus, cet infinitif a la fonction textuelle de présenter le changement de locuteur ou la reprise de parole d'un même locuteur en marquant un statut différent entre ses interventions. Voilà pourquoi nous l'avons trouvé essentiellement dans les articles de presse afin d'assurer la distribution du discours rapporté comme l'explique Englebert (1998: 79). En plus, l'infinitif de narration présente des actions réalisées par les personnages du texte ou des attitudes prises par eux.

Infinitif injonctif: Les exemples de notre corpus relèvent pour la plupart de guides de produits divers, de notices de médicaments et de recettes de cuisine. Dans le corpus, nous avons observé que les infinitifs injonctifs sont plutôt des verbes actionnels. Enfin, nous avons examiné des infinitifs injonctifs précédés de la préposition à. Notre interprétation consiste à les considérer comme des ordres à une notion d’obligation plus forte.

Infinitif interrogatif: II y en a deux types: a) des infinitifs constitués du syntagme infinitif et un pronom interrogatif et b) des infinitifs qui ne se font pas accompagner de pronom interrogatif. Les exemples du premier type sont présents dans des titres et des sous-titres de différents genres textuels du corpus. Souvent, ils se combinent avec des infinitifs injonctifs. Nous considérons que les infinitifs interrogatifs se constituent comme des stratégies textuelles pour attirer l'attention du récepteur du texte, tandis que les infinitifs injonctifs sont employés pour offrir une réponse aux interrogations posées à travers des infinitifs interrogatifs au début du fragment textuel. 
Infinitif exclamatif: Enfin, nous avons trouvé des ressemblances entre les infinitifs exclamatifs et les infinitifs interrogatifs du deuxième type. Leur but communicatif se distingue grâce au schéma prosodique à l'oral ou à la ponctuation à l'écrit. L'infinitif interrogatif du deuxième type est présent dans des textes à structure monologique ou dialogique et il exprime surtout la délibération et la perplexité, tandis que l'infinitif exclamatif est utilisé surtout dans des textes à structure dialogique afin de montrer l'indignation et la surprise par rapport à ce qui a été dit par un autre locuteur.

Après avoir exposé les résultats de notre étude, nous considérons que nous avons atteint l'objectif que nous avons défini au début : analyser les constructions infinitives indépendantes à partir d'une perspective syntaxique et textuelle. En effet, dans ce travail nous avons montré les fonctions qu'assument ces constructions au niveau de la phrase et au niveau du texte. En outre, nous considérons convenable de continuer à analyser les infinitifs indépendants avec des corpus plus vastes et selon une diversité de perspectives linguistiques qui nous permettent d'en savoir plus.

\section{Références bibliographiques}

ADAm, Jean-Michel, 2001 : «Types de textes ou genres de discours? Comment classer les textes qui disent de et comment faire? "), Langages 141, Les discours procéduraux, 10-27.

Denis, Delphine, et Anne Sancier-Chateau, 1994: Grammaire du français, Paris : Hachette.

EL HADI, Salima, 2011 : « Dans le genre 'peut mieux faire' : le bulletin scolaire », Linx 64-65, Les genres de discours vus par la grammaire, 189-206.

El KaSSAS, Dina, 2007 : «Vers une typologie des équivalents syntaxiques arabes de l'infinitif français » en Proceedings of Al Alsun International Conference.

Englebert, Annick, 1998 : L’Infinitif dit de narration, Paris, Bruxelles : Duculot.

Grevisse, Maurice, et André Goose, 2005 : Le bon usage. Grammaire française, Paris : Duculot.

Hernanz, María Llüisa, 1999: «El infinitivo » en Ignacio Bosque et Violeta Demonte (éds.) : Gramática descriptiva de la lengua española, Madrid: Espasa, 2197-2356.

Huot, Hélène, 1981 : Constructions infinitives du français : le subordonnant de, Genève-Paris : Droz.

KABATEK, Johannes, 2008 : «En primer lugar, agradecer - wie sich „ungrammatische” Infinitive in die spanische Grammatik schleichen » en Elisabeth StaRk, Roland SCHmidT-RIESE et Eva Stoll (éds.) : Romanische Syntax im Wandel, Tübingen: Narr, 197-208. 
Khodabocus, Nooreeda, 2014 : «L'infinitif : quelle catégorie? 》 en Procédés du IVème Congrès Mondial de Linguistique Française - CMLF 2014.

LABLANCHE, Anne, 2007 : L'infinitif complément d'un verbe, d'un adjectif, d'un nom : écriture d'un fragment de grammaire. Thèse non publiée. Université Paris X- Nanterre.

MelIs, Ludo, 2000 : «L'infinitif de narration comme prédication seconde », Langages 127, 36-48.

Quintero Ramirez, Sara, 2014a: «Factores pragmático-culturales del infinitivo enunciativo y del infinitif de narration en los medios de comunicación de América Latina y Canadá », FIAR: Forum for Inter-American Research 7 (1), 1-18.

Quintero Ramirez, Sara, 2014b: " Juste vous dire quelque chose à propos de l'infinitif. Étude de l'infinitif performatif d'après une perspective pragmatico-textuelle » en F. Neveu, P. BLumenthaL, L. Hriba, A. Gerstenderg, J. Meinschaefer et S. Prevost (éds.) : 4e Congrès Mondial de Linguistique Française, SHS Web of Conferences, vol. 8, 2147-2163.

Quintero Ramirez, Sara, 2015 : «Et nous de parler de l'infinitif de narration. Rasgos textuales del infinitivo narrativo » en Leonel Ruiz Mirares et al. (éds.) : Comunicación Social : Retos y Perspectivas, Santiago de Cuba : Centro de Lingüística Aplicada, 268-272.

Remi-Giraud, Sylviane, 1988 : L’infinitif, Lyon: Presses Universitaires de Lyon.

Riegel, Martin, Jean-Christophe Pellat et René Rıoul, 2001 : Grammaire méthodique du français, Paris : Presses Universitaires Françaises.

SAndfeld, Kristian, 1965 : Syntaxe du français contemporain : L’infinitif, vol. 83, Genève : Droz.

StAGE, Lilian, 2006 : «Et de conclure... sur une note optimiste! L'infinitif historique ou narratif revisité », Revue Romane 41 (1), 81-108.

TORTERAT, Fréderic, 2008 : «Citation et textologie du journal : ce que vient faire l'infinitif de narration dans les colonnes des quotidiens », Discours 3 (1) [en ligne : http://discours.revues.org/ index4343.html].

TURner, Nigel, 2000 : Linguistique contrastive et traduction, étude contrastive de l'infinitif en français et en anglais, Paris : Ophrys. 